\title{
Low-temperature thermal and elastoacoustic properties of butanol glasses: Study of position isomerism effects around the boson peak
}

\author{
Merzak Hassaine and Miguel A. Ramos* \\ Laboratorio de Bajas Temperaturas, Departamento de Fisica de la Materia Condensada, and Instituto de Ciencia de Materiales "Nicolás \\ Cabrera," Universidad Autónoma de Madrid, Cantoblanco, E-28049 Madrid, Spain
}

A. I. Krivchikov, I. V. Sharapova, and O. A. Korolyuk

B.Verkin Institute for Low Temperature Physics and Engineering of NAS of Ukraine, Kharkov, Ukraine

Rafael J. Jiménez-Riobóo

Instituto de Ciencia de Materiales de Madrid, Consejo Superior de Investigaciones Cientificas (ICMM-CSIC), Cantoblanco, E-28049 Madrid, Spain

(Received 13 December 2011; revised manuscript received 8 February 2012; published 19 March 2012)

\begin{abstract}
We have concurrently measured the specific heat, the thermal conductivity, and the longitudinal and transverse sound velocities at low temperature of glasses from different isomers of butanol ( $n$-butanol, sec-butanol and isobutanol), as well as the low-temperature specific heat for the crystals of $n$-butanol, isobutanol and tert-butanol. Whereas the elastic constants both for crystals and glasses are found to be almost independent of the position of the hydrogen bonds, the thermal properties at low temperatures of these glasses at a few kelvin (around the boson peak in the reduced specific heat or around the plateau in the thermal conductivity) are found to vary strongly. Our experiments clearly contradict other works or models claiming a Debye scaling of the boson peak, and hence of the excess low-temperature specific heat of glasses. Data analysis based upon the soft-potential model and its extensions allows us to estimate the Ioffe-Regel limit in these and other alcohol glasses, finding a correlation with the boson-peak position in agreement with that previously reported by other groups.
\end{abstract}

DOI: 10.1103/PhysRevB.85.104206

PACS number(s): $65.60 .+\mathrm{a}, 65.40 . \mathrm{Ba}, 66.70 . \mathrm{Hk}, 63.50 .-\mathrm{x}$

\section{INTRODUCTION}

Whereas the properties of crystalline solids at low temperatures are well understood within the Debye theory, the rich universal behavior of glasses and amorphous solids, especially in the temperature range $T \sim 1-20 \mathrm{~K}$ [the broad maximum in the reduced specific heat $C_{p} / T^{3}$, the corresponding "boson peak" in the reduced vibrational spectra $g(\omega) / \omega^{2}$, the plateau in the thermal conductivity $\kappa(T)$, etc. $)],{ }^{1}$ still remains a controversial issue.

Below $1 \mathrm{~K}$, the so-called tunneling model (TM) proposed 40 years ago ${ }^{1,2}$ accounts in a relatively simple way for many lowtemperature properties of amorphous solids, essentially using only two free parameters: the density of tunneling states or twolevel systems (TLS) $P_{0}$ and their coupling energy to phonons $\gamma_{L, T}$, where $L$ and $T$ stand for longitudinal and transverse phonons, respectively. Furthermore, the dimensionless ratio $C_{L, T}=P_{0} \gamma_{L, T}^{2} / \rho v_{L, T}^{2}$, where $\rho v_{L, T}^{2}$ are the corresponding elastic constants of the glass, is the ultimate single parameter that determines the even quantitatively universal behavior of noncrystalline solids at low temperatures, mainly the observed plateaus in thermal conductivity and acoustic attenuation. ${ }^{1,3}$ The fact that $C_{L, T} \sim 5 \times 10^{-4}$ (within less than one order of magnitude) for almost any glass, amorphous solid, or even disordered crystal, despite a great variation in $P_{0}, \gamma_{L, T}, \rho$, and $v_{L, T},{ }^{3}$ is the truly unexplained universality (with very few exceptions; see Ref. 4) of disordered matter. This was already emphasized by $\mathrm{Yu}$ and Leggett, ${ }^{5}$ who questioned the generally assumed picture of independent, noninteracting TLS in glasses, and even the validity of the TM itself, arguing that the universality of these low-energy excitations could be rather the result of interactions between some kind of elastic defects. Some later theoretical ${ }^{6}$ and experimental ${ }^{7,8}$ works have provided further support for these criticisms to the standard TM. Nonetheless, most of the experiments on low-temperature properties of glasses were conducted and published in the $1970 \mathrm{~s}$ and $1980 \mathrm{~s},{ }^{1}$ and interpreted within the TM.

Then, since the next decade the scientific community turned its attention to what occurs above $1 \mathrm{~K}$, and correspondingly to the glassy dynamics around $1 \mathrm{THz}$ or a few meV. The central issue of debate and considerable controversy is the so-called boson peak, ${ }^{9}$ observed as an unexpected and universal broad maximum in low-frequency Raman, inelasticneutron or inelastic-x-ray scattering, as well as in the $C_{p} / T^{3}$ representation of the specific heat around $5-10 \mathrm{~K}$, and is likely related to the above-mentioned universal plateau of the thermal conductivity in the same temperature range.

Different approaches and models have been proposed to explain those also universal properties exhibited by glasses above $1 \mathrm{~K}$, including the ubiquitous boson-peak feature. Based upon the idea that there exist in glasses additional (nonacoustic) quasilocal vibrations that can be modeled by asymmetric quartic potentials, ${ }^{10,11}$ the soft-potential model (SPM) ${ }^{12-15}$ which can be regarded as a generalization of the TM, was able to account in a consistent way for most of these glassy properties at low temperatures and low frequencies, at least up to the boson-peak energy. Further support to the SPM picture was given by the theory of vibrational instability of weakly interacting quasilocal harmonic modes. ${ }^{16,17}$ Based on more general grounds, this theory explained in a unified way the observed density of tunneling and vibrational states at 
low frequencies or temperatures [giving $g(\omega) \propto \omega^{4}$ and hence $C_{p} \propto T^{5}$, in agreement with the SPM], and also accounts for the universal appearance of a maximum in the reduced vibrational density of states (VDOS) $g(\omega) / \omega^{2}$ at slightly higher frequencies, i.e., the boson peak. A not very different and also unified picture of TLS and cooperative motions involving 200 molecules was obtained from the random first-order transition (RFOT) theory of the glass transition by Wolynes and co-workers, ${ }^{18}$ though its specific quantitative predictions for the universal low-temperature properties of glasses do not seem to have drawn much attention to experimentalists, or even been found to disagree with experiments. ${ }^{19}$

An alternative approach was taken by Schirmacher and coworkers, ${ }^{20}$ who proposed a theory for the vibrational dynamics of disordered solids based on the random fluctuation of transverse elastic constants. Later on Rufflé et al. ${ }^{21}$ presented and discussed experimental results on the VDOS and on the acoustic modes of several different glasses in the $\mathrm{THz}$ region, comparing them with the relevant predictions of the two main categories of models: harmonic random matrix models (such as that by Schirmacher) and those with additional quasilocal vibrations (in particular, the SPM). In brief, they found good agreement of experimental results only with the latter kind of model.

Very recently, Chumakov et al. ${ }^{22}$ have reported a gradual transformation of the boson peak in one sodium silicate glass into the transverse acoustic van Hove singularity of the crystalline counterpart. This experiment lends support to explanations of the boson peak in terms of mainly transverse sound waves modified by the disorder, as in the abovementioned Schirmacher model. ${ }^{20}$ Nevertheless, Chumakov et al. ${ }^{22}$ do not exclude the presence of additional quasilocalized modes in glasses, and indeed found that vibrational states at the boson peak seem to include librations of $\mathrm{SiO}_{4}$ tetrahedra.

A related issue to which much attention is being recently paid is that of the so-called Ioffe-Regel limit ${ }^{23}$ and its apparent relation to the boson-peak frequency. The IoffeRegel frequency $\omega^{\mathrm{IR}}$ is defined as the phonon frequency for which the mean free path $l$ has decreased down to half the wavelength $\lambda$ (i.e., $\omega_{L, T}^{\mathrm{IR}}=\pi v_{L, T} l_{L, T}^{-1}$, for longitudinal and transverse acoustic phonon branches, respectively). Several possible correlations between the Ioffe-Regel crossover $\omega^{\mathrm{IR}}$ and the boson peak $\omega_{\mathrm{BP}}$ have been proposed or discussed. ${ }^{24-27}$ In the above-mentioned theory of vibrational instability, the interactions between quasilocal harmonic modes and sound waves lead in a natural way to the Ioffe-Regel crossover for acoustic phonons around the boson-peak frequency. ${ }^{17}$ Although there is still no consensus even about the proper experimental data, ${ }^{25,28}$ most authors claim to find that $\omega^{\mathrm{IR}} \approx$ $\omega_{\mathrm{BP}}$, in some cases only for the transverse phonons $\omega_{T}^{\mathrm{IR}} \approx$ $\omega_{\mathrm{BP}}{ }^{27}$

In particular, glass-forming molecular liquids are interesting and appealing model systems to investigate the universal low-temperature properties of glasses, since they often allow one to control several key variables such as comparing different isotopic compositions ${ }^{29-31}$ or different isomers for the same substance, ${ }^{30,32-34}$ or using the existence of polymorphism to critically compare phases with different kinds of disorder. ${ }^{35-39}$ In addition, these molecular liquids offer an easy and convenient temperature range to explore liquid-solid phase transitions such as the glass transition, and other relaxational processes. $^{40-44}$

In this work we have conducted experiments at low temperatures with the four position isomers (also called chemical or structural isomers) of butanol, though in only three of them were we able to obtain the glass state. Also, in three out of the four cases, we were able to obtain the fully crystalline state and measured their specific heat as a useful reference. This will allow us to carry out a comparative study for different glasses of the same substance, butanol, in order to assess the effects of changing the spatial arrangement of atoms and the position of hydrogen bonds in the lattice on the low-temperature thermal and elastoacoustic properties around the universal boson-peak feature in glasses.

In the next section, we will briefly describe the materials and methods used to prepare the different samples, as well as the experimental techniques employed. Then, the experimental data directly obtained from the measurements will be shown, leaving their deeper analysis and interpretation for the Discussion section. Finally, the main conclusions of the work will be summarized.

\section{EXPERIMENTAL TECHNIQUES AND MATERIALS}

High-purity (anhydrous grade, with $<0.005 \%$ water) bottles of the different butanol chemical isomers were purchased from Sigma-Aldrich, stored in a dry glove box, and then used without further purification: $n$-butanol (also called 1-butanol; 99.8\% purity), sec-butanol (or 2-butanol; 99.5\%), tert-butanol (99.5\%), and isobutanol (99.5\%). As depicted in Fig. 1, the structure of the butanol molecule and the position of the hydroxyl group responsible for the hydrogen bonding in the solid state, are significantly different in each case.

As described in more detail elsewhere, ${ }^{39,45}$ we have implemented a calorimetric system for glass-forming liquids that allows in situ preparation and calorimetric characterization of the different phases of the substance (typically using liquid nitrogen as thermal sink), followed by accurate heat-capacity measurements at low temperatures, where the liquid-nitrogen bath is readily replaced by liquid helium, using two alternative thermal relaxation methods. ${ }^{45}$

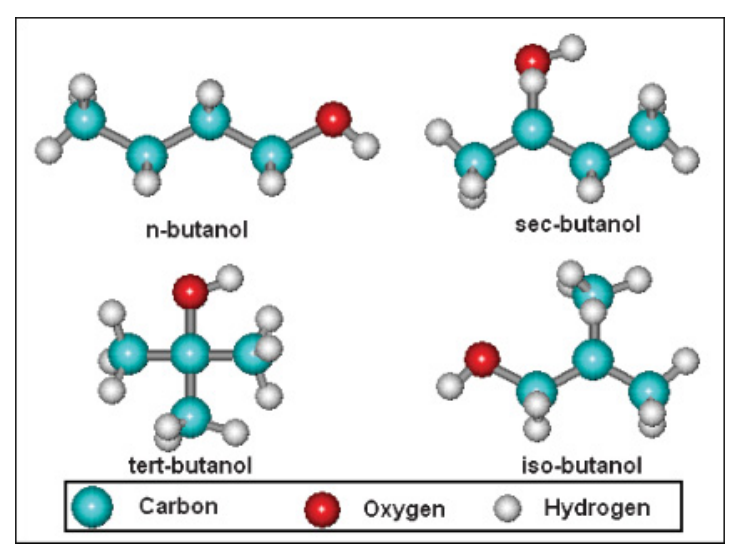

FIG. 1. (Color online) Schematic molecular structure of the four different position isomers of butanol. Note the different position of the hydroxyl group within the butanol molecular chain in each case. 
The thermal conductivity of the different position isomers of butanol was measured under equilibrium vapor pressure in an experimental setup already described, ${ }^{46}$ using the steadystate potentiometric method. The glasses were prepared by very fast cooling (above $50 \mathrm{~K} \mathrm{~min}^{-1}$ ) of the liquid through the glass-transition region.

High-resolution Brillouin-scattering measurements of both longitudinal and transverse sound velocities of the different samples in their glass state were conducted in the temperature range $10-110 \mathrm{~K}$ by using an $\mathrm{Ar}^{+}$ion laser (wavelength = $514.5 \mathrm{~nm}$ ) and a Sandercock-type $3+3$ tandem FabryPérot interferometer. The experimental setup was the same previously used and described ${ }^{47,48}$ for the study above $100 \mathrm{~K}$ of the phase diagram of $n$-butanol. In order to obtain direct information of the sound propagation velocities, the $90 \mathrm{~A}$ scattering geometry was used. ${ }^{49}$ In this way the acoustic wave vector is independent of the refractive index of the sample, and hence the sound velocities can be obtained in absolute terms. Further details on the experimental methods followed can be found in Ref. 50, where earlier experiments conducted on glassy and crystalline phases of $n$-butanol were described.

\section{RESULTS}

We will first show our overall obtained experimental data, leaving a deeper analysis for the next section. In Fig. 2, specific-heat $C_{p} / T^{3}$ plots for the achieved glass and crystal phases of the different isomers of butanol are presented. For the three crystals, the Debye coefficients can be readily obtained from a least-squares straight-linear fit (not shown) for $C_{p} / T$ vs $T^{2}$ curves at low temperatures. The obtained values are given in Table I and are graphically indicated in Fig. 2 by dashed lines. The corresponding molecular Debye temperatures $\Theta_{D}$ are also given in Table I. By molecular Debye temperatures, we mean considering in the Debye formula the number density of molecules rather than that of atoms, as is usually done, since there are only three acoustic phonon branches per primitive

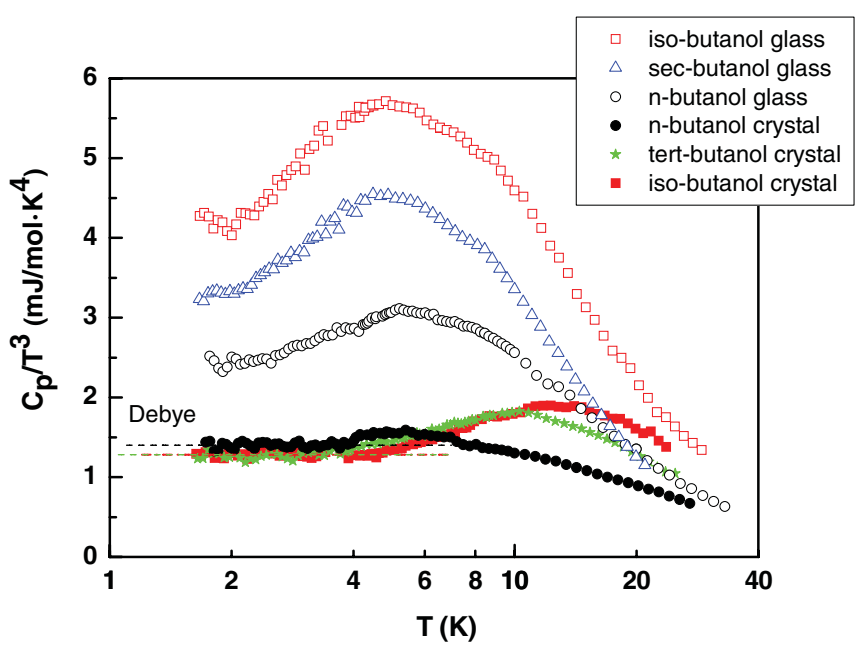

FIG. 2. (Color online) $C_{p} / T^{3}$ plots for glasses and crystalline phases of different position isomers of butanol, as indicated in the legend, showing similar Debye levels (dashed lines) for the crystals and very different boson-peak heights for the glasses.
TABLE I. Debye specific-heat coefficients $C_{\text {Debye,cal }}$ measured for crystals and corresponding molecular Debye temperatures (see text). $T_{\max }$ is the temperature of the shallow maximum in $C_{p} / T^{3}$ and $P_{c}$ is its height.

\begin{tabular}{lcccc}
\hline \hline Substance & $\begin{array}{c}C_{\text {Debye,cal }} \\
\left(\mathrm{mJ} \mathrm{mol}^{-1} \mathrm{~K}^{-4}\right)\end{array}$ & $\Theta_{D}(\mathrm{~K})$ & $T_{\max }(\mathrm{K})$ & $\begin{array}{c}P_{c} \\
\left(\mu \mathrm{J} \mathrm{g}^{-1} \mathrm{~K}^{-4}\right)\end{array}$ \\
\hline $\begin{array}{l}n \text {-Butanol } \\
\text { sec-Butanol }\end{array}$ & $1.40 \pm 0.03$ & $112 \pm 1$ & 5.4 & 21.5 \\
tert-Butanol & $1.28 \pm 0.03$ & $115 \pm 1$ & 10.1 & 24.6 \\
Isobutanol & $1.28 \pm 0.02$ & $115 \pm 1$ & 12.9 & 25.6 \\
\hline \hline
\end{tabular}

cell basis, and this appears to contain the whole molecule, not a single atom. ${ }^{51}$

In contrast to the crystals, the glasses exhibit a huge quantitative variation in the height of the $C_{p} / T^{3}$ broad maximum (i.e., the calorimetric boson peak). Whereas the Debye contributions in the crystals for $n$-butanol and isobutanol are very similar (as well as those determined for their glass states; see Table II below), the glass of isobutanol has a $C_{p} / T^{3}$ peak height which doubles the one of $n$-butanol. Also, the glass of sec-butanol (2-butanol) presents a $C_{p} / T^{3}$ peak that is $50 \%$ higher than the peak of $n$-butanol (1-butanol). This finding resembles what was observed for 1- and 2-propanol. ${ }^{30,32}$ However, in the isomers of propanol this effect was traced back to noticeable changes produced by the different hydrogenbonding arrangement into the lattice dynamics, since the Debye contributions in the corresponding crystalline states presented an analogous big change.

The temperature dependences of the thermal conductivity $\kappa(T)$ for the three obtained glass isomers of butanol are shown in Fig. 3, using a log-log plot. It can be seen that the temperature behavior of the thermal conductivity for all the butanol isomers is that typical of glasses. First, the thermal conductivity of all alcohols increases with increasing temperature (most intensively at the lowest temperatures) up to a maximum value. As expected, each curve has a smeared plateau around 5-10 K where the thermal conductivity practically does not vary with rising temperature. Then it continues to increase up to a saturation at $T \approx 50 \mathrm{~K}$, that essentially persists toward the glass-transition temperature $\left(T_{g} \sim 110-20 \mathrm{~K}\right.$ in the three cases).

It is noteworthy that the trend for the low-temperature thermal conductivity curves $\kappa(T)$ for these three isomeric glasses of butanol does not follow the corresponding (inverse) order of low-temperature specific heat $C_{p}(T)$ curves and the boson peak in $C_{p} / T^{3}$, as could be expected in principle. Indeed, under the SPM which includes the TM, the thermal conductivity is essentially controlled by the resonant scattering of sound waves by tunneling states (below $\sim 1 \mathrm{~K}$ ) or by the soft modes producing the boson peak (for the plateau region). ${ }^{13,15}$ Hence, the larger the density of tunneling states and soft modes contributing to the specific heat, the lower the thermal conductivity, if the material parameters are kept similar. Therefore, this unexpected finding puts a demanding challenge to any theory or model aiming to account for the low-temperature universal properties of glasses, especially because we are addressing a direct comparison in the very 
TABLE II. Elastic data obtained for glasses: $\rho_{\mathrm{RT}}$ are mass-density values in the liquid state at $20^{\circ} \mathrm{C} ; \rho_{0 \mathrm{~K}}$ are extrapolated values to low temperature using Eq. (2); $v_{L}(0)$ and $v_{T}(0)$ are measured longitudinal and transverse sound velocities in the zero-temperature limit from Fig. 4 , and $v_{D}$ is their Debye-averaged sound velocity; $C_{\text {Debye }}$ is the predicted Debye cubic coefficient for the specific heat and $\Theta_{D}$ is the corresponding molecular Debye temperature, after Eq. (1); $T_{\max }$ is the temperature of the boson-peak maximum in $C_{p} / T^{3}$ and $P_{c}$ is its height.

\begin{tabular}{|c|c|c|c|c|c|c|c|c|c|}
\hline Substance & $\begin{array}{c}\rho_{\mathrm{RT}} \\
\left(\mathrm{kg} / \mathrm{m}^{-3}\right)\end{array}$ & $\begin{array}{c}\rho_{0 \mathrm{~K}} \\
\left(\mathrm{~kg} / \mathrm{m}^{-3}\right)\end{array}$ & $\begin{array}{l}v_{L}(0) \\
(\mathrm{m} / \mathrm{s})\end{array}$ & $\begin{array}{l}v_{T}(0) \\
(\mathrm{m} / \mathrm{s})\end{array}$ & $\begin{array}{c}v_{D} \\
(\mathrm{~m} / \mathrm{s})\end{array}$ & $\begin{array}{c}C_{\text {Debye }} \\
\left(\mathrm{mJ} \mathrm{mol}^{-1} \mathrm{~K}^{-4}\right)\end{array}$ & $\Theta_{D}(\mathrm{~K})$ & $T_{\max }(\mathrm{K})$ & $\begin{array}{c}P_{\mathrm{c}} \\
\left(\mu \mathrm{J} \mathrm{g}^{-1} \mathrm{~K}^{-4}\right)\end{array}$ \\
\hline$n$-Butanol & 809.5 & 951.0 & 3144 & 1572 & $1763 \pm 18$ & $1.74 \pm 0.09$ & $104 \pm 2$ & 5.4 & 42.0 \\
\hline sec-Butanol & 806.3 & 947.6 & 3070 & 1550 & $1738 \pm 17$ & $1.82 \pm 0.09$ & $102 \pm 2$ & 4.8 & 61.4 \\
\hline tert-Butanol & 780.9 & & & & & & & & \\
\hline Isobutanol & 802 & 908.5 & 3119 & 1521 & $1709 \pm 17$ & $2.00 \pm 0.1$ & $99 \pm 2$ & 4.8 & 77.0 \\
\hline
\end{tabular}

same experimental conditions and for the same chemical composition.

These results should also be compared to those previously obtained in propanol. ${ }^{34}$ In that case, a very large plateau in the thermal conductivity was observed for 1-propanol at $T=$ 6-90 K, including a deflection at $T=20 \mathrm{~K}$. In 2-propanol, the plateau occurred in a much narrower temperature interval (5-10 K). Among the butanol isomers, the largest plateau is observed in $n$-butanol $(5-12 \mathrm{~K})$, is smaller in isobutanol $(5-10 \mathrm{~K})$, and has a lower value in sec-butanol $(5-8 \mathrm{~K})$. The most pronounced variations in the thermal conductivity of these isomers is observed at low temperatures: at $T=$ $2 \mathrm{~K}$ the thermal conductivity of 1-propanol was 1.6 times higher than that of 2-propanol. ${ }^{34}$ The butanol glasses behave similarly: the thermal conductivity of $n$-butanol (1-butanol) is almost 1.5 times higher than the corresponding values for sec-butanol (2-butanol); isobutanol shows an intermediate magnitude. At $T \geqslant 10 \mathrm{~K}$ the difference in the thermal conductivity decreases gradually, and from $T \geqslant 40 \mathrm{~K}$ up to the glass-transition temperature $T_{g}$ the position isomerism has no effect on the thermal conductivity of the considered alcohols: all curves merge into a single one, which exhibits a saturation around $50 \mathrm{~K}$.

Our measurements of hypersonic longitudinal and transverse sound velocities, as a function of temperature $v_{L, T}(T)$ for

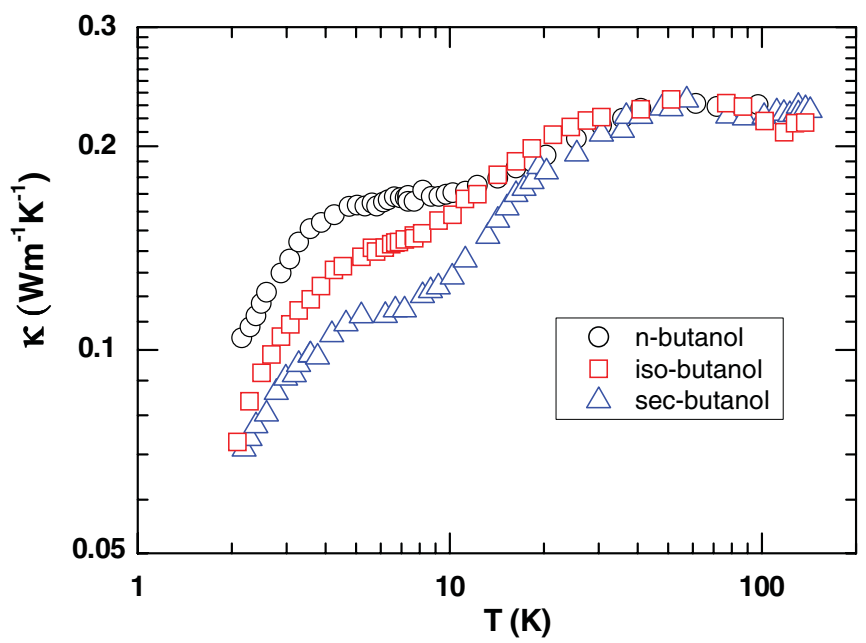

FIG. 3. (Color online) Temperature dependence of the thermal conductivity of $n$-butanol (circles), sec-butanol (squares), and isobutanol (triangles), in their glass states. the three butanol glasses below their glass-transition temperatures down to $10 \mathrm{~K}$, are shown in Fig. 4. Simple extrapolations to zero temperature $v_{L, T}(T=0)$, used to determine the elastic Debye coefficients and Debye temperatures through

$$
C_{\text {Debye }}=\frac{2 \pi^{2}}{5}\left(\frac{k_{B}^{4}}{\hbar^{3} \rho v_{D}^{3}}\right)=234\left(\frac{N k_{B}}{\rho \Theta_{D}^{3}}\right),
$$

where $v_{D}$ is the Debye-averaged sound velocity $1 / v_{D}^{3}=$ $1 / 3\left(1 / v_{L}^{3}+2 / v_{T}^{3}\right)$, are also graphically indicated in the figure and given in Table II. $N$ is taken as the number density of molecules for the reasons given above. The Debye sound velocity values $v_{D}$ found for the three position isomers of butanol are very similar, ranging $1709-1763 \mathrm{~m} / \mathrm{s}$.

In order to assess the values of the mass density in the zerotemperature limit $\rho_{0 \mathrm{~K}}$, we have obtained the ratios $\rho_{R T} / \rho_{0 \mathrm{~K}}$ between their values in the liquid state at room temperature $\left(20^{\circ} \mathrm{C}\right)$ taken from the literature and those unknown at $0 \mathrm{~K}$ by assuming that the Lorentz-Lorenz specific refraction

$$
r=\frac{n^{2}-1}{n^{2}+2} \frac{1}{\rho}
$$

remains constant ${ }^{52}$ as a function of temperature. From our Brillouin-scattering measurements we have obtained the refractive index both at room temperature and at low temperatures, and hence the corresponding extrapolated values $\rho_{0 \mathrm{~K}}$ for the three glassy isomers, which are given in Table II. The errors involved in these estimations (obtained from typical propagation of error analysis, and indicated in the tables) are deemed not to be very relevant for the following discussions. The correspondingly determined values of the predicted Debye cubic coefficient $C_{\text {Debye }}$ for the specific heat at low temperatures and the molecular Debye temperatures $\Theta_{D}$ are also displayed in Table II.

\section{DISCUSSION}

In this section, we will discuss the presented collection of experimental results on the different glasses of butanol isomers.

\section{A. Specific heat}

First of all, the experimental data on the low-temperature specific heat and thermal conductivity for the different butanol glasses will be assessed and interpreted within the basic SPM ${ }^{13-15}$ which allows a rather straightforward comparison 

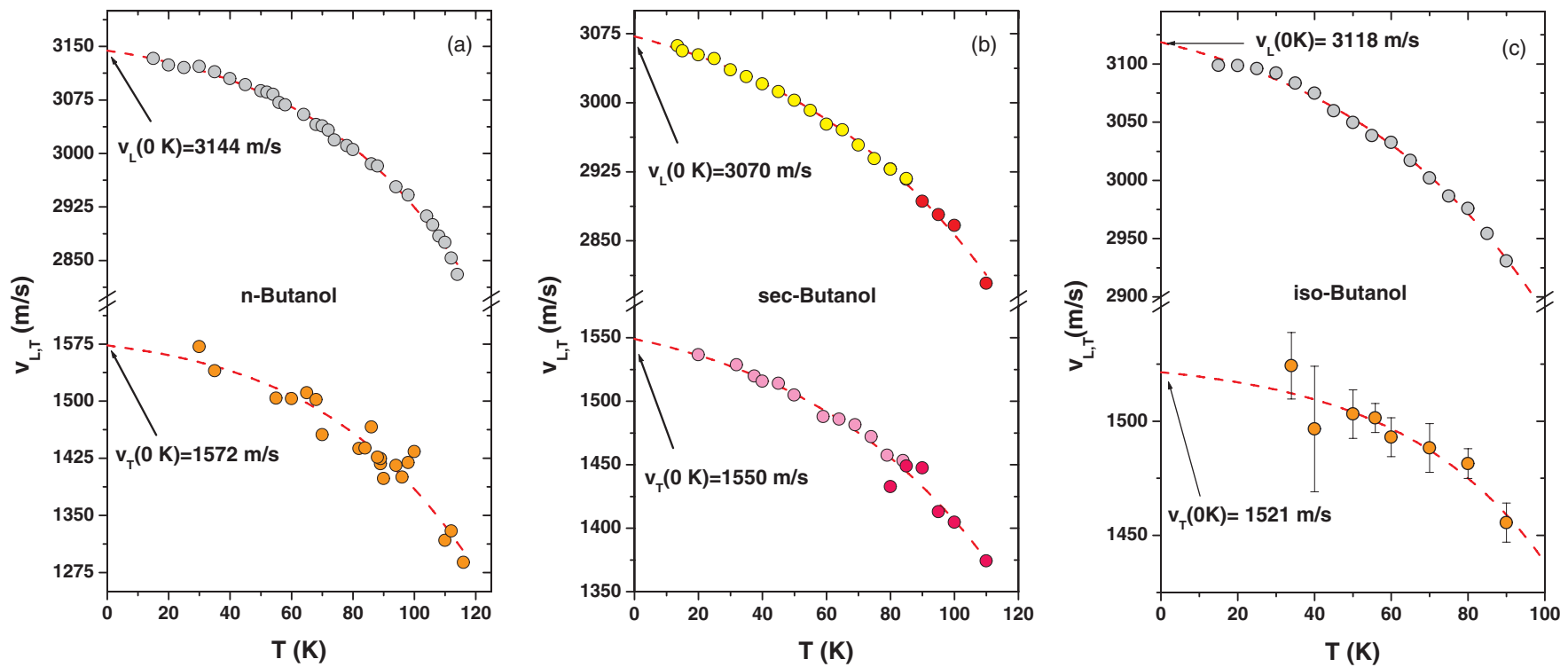

FIG. 4. (Color online) Temperature dependence (including extrapolation to zero temperature) for the longitudinal and transverse sound velocities of $n$-butanol, sec-butanol, and isobutanol isomers, in their glass states. Experimental error bars are only shown for the case of transverse sound in isobutanol, which is the one with the largest errors.

with the experimental data, and putting aside for the moment other variants or extensions of the SPM to account for the boson peak and other glassy properties at moderately high temperatures.

In the basic SPM, it is assumed that low-frequency dynamics of glasses is driven by the coexistence of usual acoustic (phononlike) lattice vibrations and some additional quasilocalized vibrations (or "soft modes") that can be described by asymmetric quartic potentials such as $V(x)=W\left(D_{1} x+D_{2} x^{2}+x^{4}\right)$, where $x$ is a generalized, dimensionless coordinate, and all the soft modes are characterized on average by a single energy $W .{ }^{15}$ The (flat-bottomed) anharmonic quartic potential $V(x)=W x^{4}$ marks the crossover from extremely anharmonic tunneling states or TLS in double-well potentials with negative quadratic coefficients $D_{2}$ to quasiharmonic vibrations in single-well potentials with positive $D_{2}$ coefficients. Similarly to the TM, the SPM postulates that the coefficients of the soft potentials, $D_{1}$ and $D_{2}$, are randomly distributed around those of the central quartic potential: $P\left(D_{1}, D_{2}\right)=P(0,0) \equiv P_{s}$.

Under those simple assumptions, the specific heat is found to be approximately linear ${ }^{14,15}$ in temperature for $k_{B} T \ll$
$W$ due to the dominant contribution of the TLS, therefore including most of the predictions of the TM for $T<1 \mathrm{~K}$, though with slightly different parameters and formulas. In the typical $C_{p} / T^{3}$ plot, that lowest-temperature TLS region is followed by a minimum and a rapid increase produced by the quasiharmonic soft-mode vibrations with a density $g_{\text {sm }}(\omega) \propto \omega^{4}$ (i.e., the lower-energy tail of the boson peak) and a corresponding specific-heat contribution ${ }^{15}$

$$
C_{\mathrm{sm}}=\frac{2 \pi^{6}}{21} P_{s} k_{B}\left(\frac{k_{B} T}{W}\right)^{5}
$$

At and above the boson-peak maximum, these simple analytical approximations fail. In order to analyze quantitatively the specific-heat data of glasses through the SPM, it has been suggested ${ }^{30,53}$ that the most reasonable fit of low-temperature specific-heat data is therefore a quadratic polynomial fit in a $C_{p} / T$ vs $T^{2}$ plot to the SPM prediction

$$
C_{p}=C_{\mathrm{TLS}} T+C_{D} T^{3}+C_{\mathrm{sm}} T^{5}
$$

where the coefficients essentially correspond to the contributions of the TLS, Debye lattice vibrations, and quasiharmonic

TABLE III. SPM-fitted specific-heat parameters for glasses: $W$ and $\bar{C}$ have been determined to concurrently fit low-temperature thermal conductivity and specific-heat data; the ratio $C_{\mathrm{TLS}} / C_{\mathrm{sm}}$ is fixed from $W$; the density of soft modes $P_{s}$ is directly derived from $C_{\mathrm{sm}} . E_{\mathrm{IR}}$ is the Ioffe-Regel energy, obtained from Eq. (7).

\begin{tabular}{lcccccc}
\hline \hline Substance & $\begin{array}{c}W / k_{B} \\
(\mathrm{~K})\end{array}$ & $\begin{array}{c}C_{\mathrm{TLS}} \\
\left(\mathrm{mJ} \mathrm{mol}^{-1} \mathrm{~K}^{-2}\right)\end{array}$ & $\begin{array}{c}C_{\mathrm{sm}} \\
\left(\mathrm{mJ} \mathrm{mol}^{-1} \mathrm{~K}^{-6}\right)\end{array}$ & $\begin{array}{c}P_{s} \\
\left(\mathrm{~mol}^{-1}\right)\end{array}$ & $\begin{array}{c}\bar{C} \\
\left(\times 10^{-4}\right)\end{array}$ & $\begin{array}{c}E_{\mathrm{IR}} / k_{B} \\
(\mathrm{~K})\end{array}$ \\
\hline $\begin{array}{l}n \text {-Butanol } \\
\text { sec-Butanol }\end{array}$ & 2.7 & 0.86 & 0.106 & $1.20 \times 10^{19}$ & 2.25 & 41.4 \\
tert -Butanol & 2.8 & 2.11 & 0.225 & $3.06 \times 10^{19}$ & 3.6 & 36.7 \\
Isobutanol & 3.1 & 4.13 & 0.293 & $6.64 \times 10^{19}$ & 3.5 & 41.0 \\
\hline \hline
\end{tabular}


soft modes, respectively. To be meaningful, this fit should be performed ${ }^{30,53}$ approximately in the temperature range $0<T<(3 / 2) T_{\min }$, where $T_{\min }$ is the temperature of the minimum value of $C_{p} / T^{3}$.

Nonetheless, for an accurate determination of those SPM parameters (especially the TLS coefficient) in our case, measurements at still lower temperatures would be needed, since the TLS- soft modes crossover for the specific heat occurs approximately between $k_{B} T_{\min } \approx W / 1.6\left(\right.$ Ref. 12) and $k_{B} T_{\min } \approx$ $W / 1.8,{ }^{15}$ which is around or below $2 \mathrm{~K}$ in this case, too close to the lowest temperatures in our experiments. A similar problem affects the thermal conductivity data. The crossover from the lowest-temperature region dominated by the resonant scattering of sound waves by tunneling states $\left(\kappa \propto T^{2}\right)$ to that by soft modes (the plateau region) can be assessed from a plot of $\kappa / T$ vs $T$, where a maximum is observed at $T_{\max , \kappa}$ given by $k_{B} T_{\max , \kappa} \approx W / 1.6{ }^{15}$ Therefore, rather than trying separate best fits for every measurement (probably inconsistent for the reasons given above), we will determine a single $W$ SPM parameter for a given substance, taking a compromise between both thermal properties. Then, the corresponding quantitative factors $\left[C_{\mathrm{TLS}}, C_{\mathrm{sm}}\right.$, and the averaged dimensionless ratio $\bar{C}$ defined below by Eq. (6)] are fitted to scale the corresponding data (see Table III).

Also for the lack of specific-heat data at very low temperatures and to avoid too many doubtful SPM parameters, a more reasonable and accurate procedure will be followed here than a direct fit to Eq. (4). Since we have obtained the true Debye coefficient $C_{\text {Debye }}$ from Brillouin measurements (Table II), and the elastic Debye coefficients have been proved ${ }^{54}$ to be equal to the calorimetrically obtained ones $C_{D}$, when properly fitted through Eq. (4), we have fixed the measured $C_{D}$ coefficient for each glass. The excess specific heat of the butanol glasses is hence clearly manifested after subtraction of the corresponding Debye coefficient, and plotted as $\left(C_{p}-C_{\text {Debye }}\right) / T$ vs $T^{4}$, in order to obtain the missing $T$ and $T^{5}$ coefficients of the SPM from simple straight-linear fits, as shown in Fig. 5(a) and in Table III. In those fits, the SPM parameters $C_{\mathrm{TLS}}$ and $C_{\mathrm{sm}}$ were in fact fixed to fulfill the above-mentioned rule $W / k_{B} \approx$ $(1.6-1.8) T_{\min }=(1.6-1.8)\left(C_{\mathrm{TLS}} / C_{\mathrm{sm}}\right)^{1 / 4}$. Let us stress that the observed linear behavior in the $\left(C_{p}-C_{\text {Debye }}\right) / T$ vs $T^{4}$ plots for $0<T^{4}<\left(W / k_{B}\right)^{4}$ is indeed a nontrivial confirmation of the SPM qualitative predictions.

In Fig. 5(b), we show the specific-heat data $C_{p} / T^{3}$ of the three butanol glasses together with the SPM fit below the boson peak, which is the sum of acoustically measured Debye coefficients for the glasses (dashed lines) and the SPM fitted contributions from Fig. 5(a), according to Eq. (4). These SPM fits are not expected to be very accurate because of the abovementioned difficulty due to the lack of very low temperature data.

Figure 5(b) could be compared to what was found in 1- and 2-propanol, where the different boson peaks found in them were correlated to the very different Debye levels between 1- and 2-propanol observed in both crystalline and glassy states. ${ }^{30,32}$ In clear contrast to the case of propanol, the notable variation found in the boson-peak amplitudes among different butanol isomers coexists with very similar Debye coefficients in all of them. This clear finding opposes a one-parameter
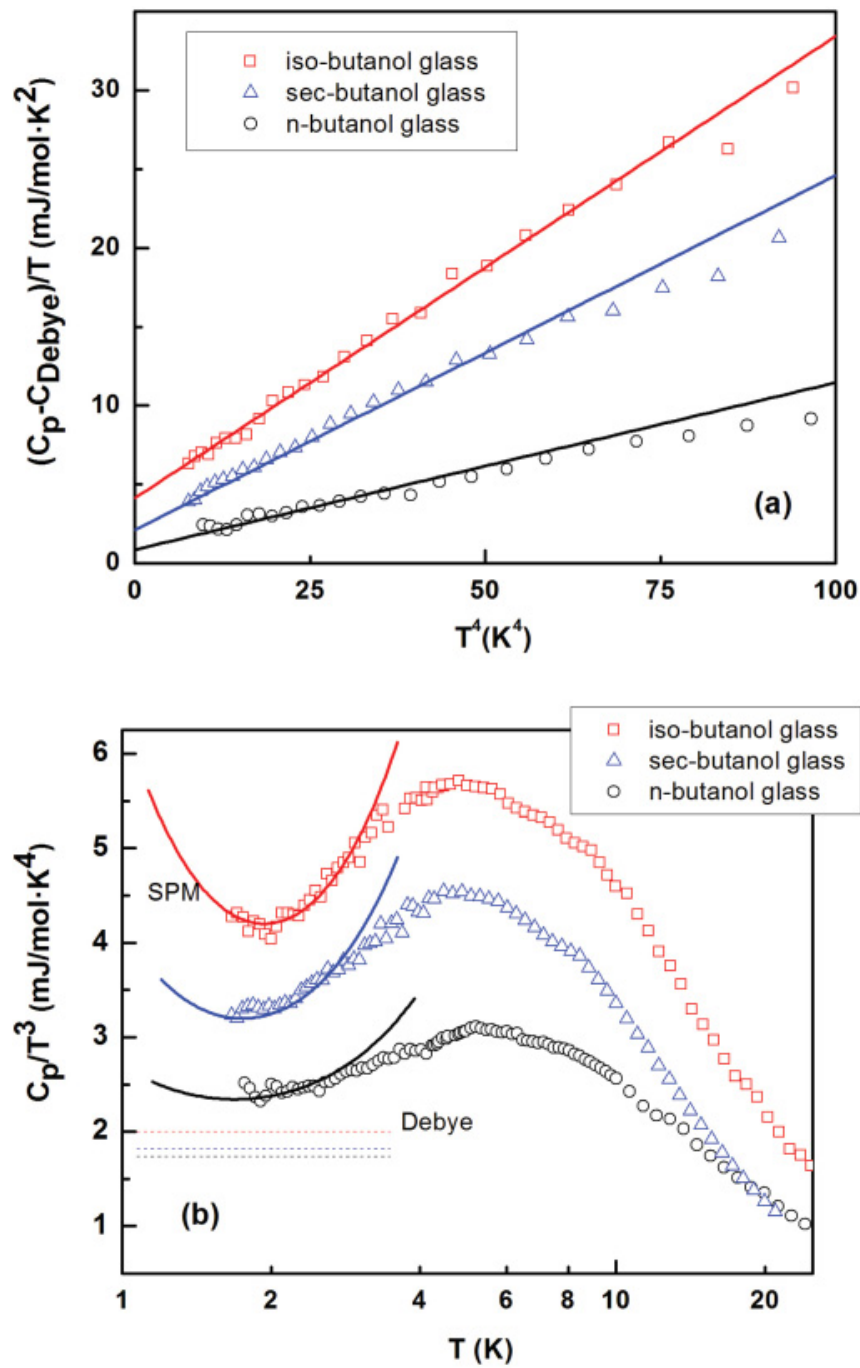

FIG. 5. (Color online) (a) Excess low-temperature specific heat of butanol glasses after subtraction of the acoustically measured Debye level in a $\left(C_{p}-C_{\text {Debye }}\right) / T$ vs $T^{4}$ plot, in order to obtain the $T$ and $T^{5}$ coefficients of the soft-potential model from the shown straightlinear fits (see text for more details). (b) $C_{p} / T^{3}$ plots for the butanol glasses. Dashed lines indicate their (similar) Debye contributions obtained from Brillouin-scattering measurements (see Fig. 4): iso-, sec-, and $n$-butanol, from above to below. The corresponding boson peaks follow the same order, but in contrast vary strongly. Solid lines indicate the total SPM fit [i.e., the one from (a) plus the Debye contribution, according to Eq. (4)].

scaling of the boson peak in glasses with (mainly transverse) acoustic phonon dispersion curves and Debye frequencies, as reported by some authors. ${ }^{22,55-57}$ As shown here, this cannot be a universal rule. The different amorphous networks built up from differently hydrogen-bonded butanol molecules produce significantly different densities of quasilocalized vibrations at low frequencies and hence boson-peak heights, whereas the acoustic properties and the Debye contribution remain practically the same. It is important to emphasize that these conclusions are independent of the conducted SPM quantitative analysis or fits, but are clearly deduced from the experiments. 
As an alternative approach to discuss the debated correlation between the boson peak and the elastic constants, we can take into account the interstitialcy model proposed by Granato. ${ }^{58,59}$ According to this model, liquids can be considered as crystals containing a few interstitials in thermal equilibrium, which become frozen in the glassy state. Those self-interstitial resonant modes could be the physical realization of the quasilocalized vibration (soft) modes and the tunneling states of the SPM. After some approximations, taking a single frequency for the resonant modes, this model even makes a quantitative prediction ${ }^{59}$ for such a correlation: $T_{\max } \sim \Theta_{D} / 35$, where $\Theta_{D}$ should be the usual atomic Debye temperature. Several authors have independently checked this proposed correlation for different collections of glasses, finding empirically $T_{\max } \sim \Theta_{D} / 40$ (Ref. 60) or even $T_{\max } \sim$ $\Theta_{D} / 35$ for some glassy alcohols as normal and deuterated ethanol, 1-propanol, and glycerol. ${ }^{53}$ Nonetheless, $T_{\max } \sim$ $\Theta_{D} / 42$ was found for 2-propanol, and $T_{\max } \sim \Theta_{D} / 46$ and $T_{\max } \sim \Theta_{D} / 49$ for strong glass formers such as $\mathrm{SiO}_{2}$ and $\mathrm{B}_{2} \mathrm{O}_{3}$, respectively. ${ }^{53}$ In our case, from the data shown in Table II (and multiplying $\Theta_{D}$ by $15^{1 / 3}$ to get the atomic Debye temperature), we obtain proportionality factors of $1 / 47$, $1 / 52.5$, and $1 / 51$ for $n$-, sec- and isobutanol, respectively. Given the expected intrinsic scaling among all those elastic energies and temperatures in a solid, such variations between different glassy substances are beyond a reasonable data scatter to justify that model. Furthermore, if we try to apply the same normalization to the shallow $C_{p} / T^{3}$ maxima of the butanol crystals (see Table I), we obtain $T_{\max } \sim \Theta_{D} / 51, T_{\max } \sim$ $\Theta_{D} / 28$, and $T_{\max } \sim \Theta_{D} / 22$ for $n$-, tert- and isobutanol, respectively.

Also, Liu and von Löhneysen ${ }^{61}$ proposed years ago a general correlation between the position $T_{\max }$ and the height $P_{c}$ of the maximum in $C_{p} / T^{3}$, finding $P_{c} \propto T_{\max }^{-1.6}$. This relation was claimed to be valid not only for all the amorphous solids found in the literature, but also for many of the corresponding crystalline solids, hence suggesting a general correlation between the mechanisms leading to the $C_{p} / T^{3}$ maxima in amorphous and crystalline solids. Since they plotted $P_{c}$ in units of $\mu \mathrm{J} \mathrm{g}^{-1} \mathrm{~K}^{-4}$, we have given the observed values for the height of the $C_{p} / T^{3}$ maxima for our crystals (Table I) and for our glasses (Table II) in the same units, in order to facilitate the comparison with that paper. All these values would lie in the above-right side of their log-log plot (i.e., Fig. 2 of Ref. 61). More importantly, it is straightforward to see that neither glassy nor crystalline butanol isomers follow the proposed $P_{\mathrm{c}} \propto T_{\max }^{-1.6}$ correlation at all, and even show opposite trends.

\section{B. Thermal conductivity}

For an elastic continuum such as a glass, the thermal conductivity $\kappa(T)$ is given by the standard expression obtained from the well-known phonon-gas kinetic equation, and using the Debye approximation for the density of states of the sound waves transporting heat. First of all, we will again analyze our thermal conductivity results within the SPM, ${ }^{13-15}$ in which the inverse mean free phonon path can be subdivided into three components describing sound-wave resonant scattering by tunneling states and quasilocalized low-frequency vibrations, as well as by classical relaxation processes:

$$
\begin{aligned}
l_{\mathrm{SPM}}^{-1}(\omega)= & 1.1 \frac{\pi \omega C_{L, T}}{v_{L, T}} \tanh \left(\frac{\hbar \omega}{2 k_{B} T}\right) \\
& +\frac{\pi \omega C_{L, T}}{v_{L, T}}\left[\left(\frac{T}{W}\right)^{3 / 4} \ln ^{(-1 / 4)}\left(\frac{1}{\omega \tau_{0}}\right)+\frac{1}{8}\left(\frac{\hbar \omega}{W}\right)^{3}\right]
\end{aligned}
$$

for longitudinal $(L)$ and transverse $(T)$ phonons, respectively. In addition to the two SPM parameters $W$ and $P_{S}$ defined above, for thermal conductivity and acoustic properties the strength parameter $C_{L, T}$ (in turn linearly proportional to $\left.P_{S} / W\right)$ is introduced instead of $P_{s}$ and appears explicitly in the equations. $C_{L, T}$ is essentially the same tunneling strength parameter defined in the TM characterizing the interaction between the acoustic sound waves and the two-level systems, but now generalized to all quasilocalized excitations within the SPM. To be more precise, $C_{L, T}^{(\mathrm{TM})} \sim 1.1 C_{L, T}^{(\mathrm{SPM})}$ was found. ${ }^{15}$ As said above, it is the astonishing universality of $C_{L, T}\left(\sim 5 \times 10^{-4}\right)$ that remains the key question concerning the low-temperature universal properties of glasses.

To compare with experiments, the thermal conductivity of the SPM can be calculated using the following expression: ${ }^{15}$

$$
\kappa(T)=\frac{2 k_{B}}{3 \pi \bar{C}}\left(\frac{W}{h}\right)^{2}\left(\frac{1}{v_{l}}+\frac{2}{v_{t}}\right) F(z) \equiv \frac{2 k_{B}}{\pi h^{2}} \frac{W^{2}}{\bar{C} \bar{v}} F(z),
$$

where $z=\frac{k_{B} T}{W}, h$ is the Planck constant, $\frac{1}{\bar{v}} \equiv \frac{1}{3}\left(\frac{1}{v_{l}}+\frac{2}{v_{t}}\right)$, $\bar{C}$ is the properly averaged value of $C_{L}$ and $C_{T}$ (which are nonetheless expected to be very similar), and

$$
F(z)=\int_{0}^{\infty} d x \frac{x^{3} e^{-x}}{\left(1-e^{-x}\right)^{2}} \frac{z^{2}}{1.1 \tanh (x / 2)+0.7 z^{3 / 4}+x^{3} z^{3 / 8}}
$$

The function $F(z)$ is hence universal and dimensionless, and only depends on the normalized temperature $z=\frac{k_{B} T}{W}$. In Ref. 15, a good agreement was found between those SPM equations and the thermal conductivity data found in the literature for several glasses at low temperatures.

According to the SPM prediction, the magnitude $\kappa(T) \frac{\pi}{2 k_{B}}\left(\frac{h}{W}\right)^{2} \bar{C} \bar{v}$ as a function of $\frac{k_{B} T}{W}$ [i.e., $F(z)$ as a function of $z$ ] should be independent of a particular substance structure or chemical composition, at least at low temperatures up to the plateau region. The SPM parameters $W$ and $\bar{C}$ can thus be obtained through a comparison of experimental data and the universal dependence of $F(z)$ given by Eq. (6). The dependence of the renormalized thermal conductivity on the reduced temperature $\left(z=\frac{k_{B} T}{W}\right)$ obtained for our experimental data in the different isomers is shown in Fig. 6. Fitting parameters $W$ and $\bar{C}$ are given in Table III. As said above, a single $W$ has been determined for a given substance as the best compromise to agree with both specific heat data and thermal conductivity data, specifically to agree with $F(z)$ for $z<2$. Then $\bar{C}$ is just an overall constant factor for the thermal conductivity curve. It can be observed indeed in Fig. 6 that the universal behavior proposed by the SPM renormalization agrees well with experimental data in the low-temperature region $(z<2)$ for the three butanol isomers, as was also observed in other structural and orientational glasses. ${ }^{62,63}$ Obviously, this SPM scaling does not work above, say, $z>4$, 


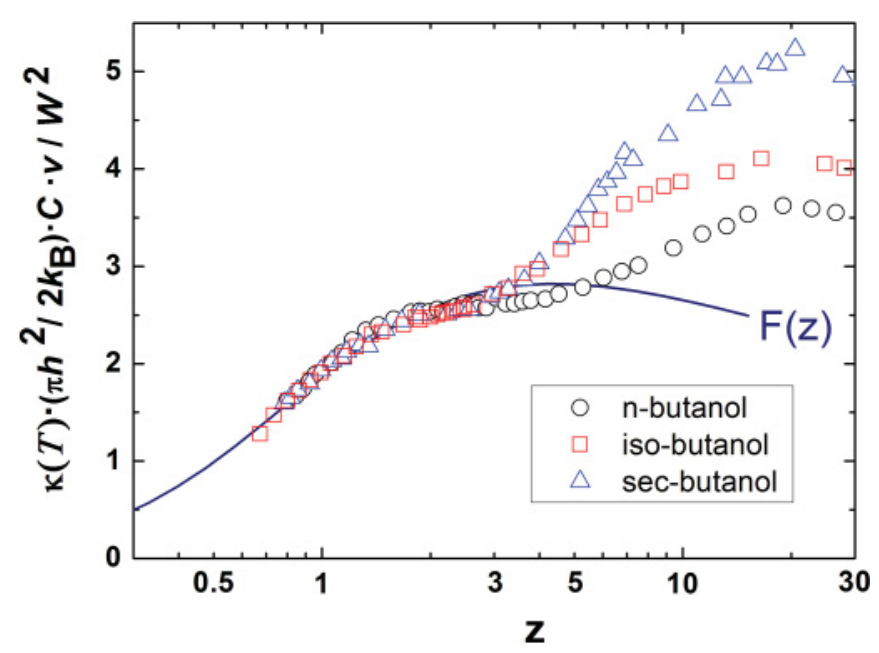

FIG. 6. (Color online) Renormalized thermal conductivity data $\kappa(T) \frac{\pi}{2 k_{B}}\left(\frac{h}{W}\right)^{2} \bar{C} \bar{v}$ plotted on the reduced temperature $z=\frac{k_{B} T}{W}$ from experimental data for butanol glasses (symbols as indicated in the legend). The solid line is the universal dimensionless function $F(z)$ (see text).

where the basic SPM is no longer valid and the similar obtained values of thermal conductivity at higher temperatures for all butanol glasses (see Fig. 3) would give different deviations from the universal SPM curve $F(z)$.

\section{Phonon mean free path}

Because of the strong frequency dependence of the inverse phonon relaxation time $l_{\mathrm{SPM}}^{-1}(\omega)$ due to the resonant scattering of acoustic modes by quasilocalized modes, the phonon mean free path reduces sharply as phonon frequency increases and reaches the Ioffe-Regel localization criterion (crossover) for phonons at the frequency $\omega_{\mathrm{IR}}$, as briefly discussed in Sec. I. According to the SPM, the plateau in the thermal conductivity curve is the result of resonant phonon scattering by soft modes but is not directly due to phonon localization.

Above the frequency $\omega_{\text {IR }}$ in the region of phonon localization the concept of phonons (acoustic modes) becomes meaningless in glasses. Acoustic modes and quasilocalized vibrations would become mixed excitations and could not be considered $^{17}$ any longer as independent of each other. This causes a rearrangement of the density of low-frequency vibrational states, ${ }^{17}$ eventually producing a maximum in $C_{p}(T) / T^{3}$ (the boson peak) at $T_{\max } \approx E_{\mathrm{IR}} / 5 k_{B}$ (where $E_{\mathrm{IR}}=\hbar \omega^{\mathrm{IR}} \approx$ $\left.\omega_{\mathrm{BP}}\right)$ and only a slight growth of the thermal conductivity with temperature. Within the SPM, one finds ${ }^{21}$

$$
E_{\mathrm{IR}}=\hbar \omega^{\mathrm{IR}}=2\left(\pi^{2} \bar{C}\right)^{-1 / 3} W .
$$

Cahill and Pohl (CP) ${ }^{64}$ have proposed a model to account for the lowest limit of the thermal conductivity observed in disordered solids. In this model, high-frequency vibrational excitations can hop from one oscillator to another just traveling a distance equal to half the wavelength $\lambda$. They found that the phonon mean free path cannot be lower than

$$
l_{\mathrm{CP}}=\pi v_{D} \omega^{-1} .
$$

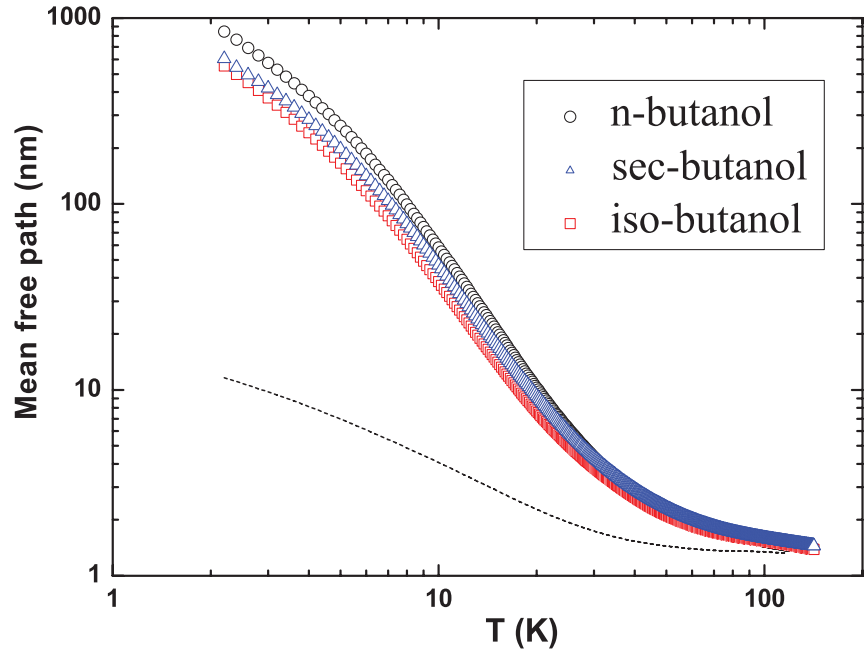

FIG. 7. (Color online) Phonon mean free paths $\ell(T)$ for the three isomers of butanol calculated from thermal-conductivity data via the standard equation of the phonon-gas kinetic theory: $n$-butanol (black circles), sec-butanol (blue triangles), and isobutanol (red squares). The dashed line is $\ell_{\min }(T)$ calculated according to the Cahill-Pohl model for isobutanol glass.

We will make use of this proposed lower bound for the minimum mean free path of phonons, and compare it to our experimental data, below in Fig. 7.

In the theory of vibrational instability, ${ }^{16,17}$ the inverse phonon mean free path $l^{-1}(\omega)$ is proportional to the density of vibrational states, $l^{-1}(\omega) \propto g(\omega)$, presenting the Ioffe-Regel crossover at a frequency $\omega^{\mathbb{I R}}$ which is very close to that of the maximum at $\omega_{\mathrm{BP}}$ in the reduced vibrational density of states $g(\omega) / \omega^{2}$. At low frequencies $\left(\omega \ll \omega^{\mathrm{IR}}\right), g(\omega) \sim \omega^{4}$ in agreement with the basic SPM, whereas at high frequencies $\left(\omega \gg \omega^{\mathrm{IR}}\right)$ it is found $g(\omega) \propto \omega \cdot{ }^{17}$ Hence a similar behavior is observed in $l^{-1}(\omega)$ : at low frequencies, $l^{-1}(\omega) \propto \omega^{4}$, where $l^{-1}(\omega)$ also depends on both $\bar{C}$ and $W$ [indeed $l^{-1}(\omega)$ increases with $\bar{C}$ and strongly decreases with $W]$; at high frequencies, there is a crossover from weak phonon scattering to strong phonon scattering at $\omega^{\mathrm{IR}}$ which corresponds to the boson peak. In this higher frequency region $l^{-1}(\omega) \propto \omega$ and depends only from the elastic properties of glass. At least qualitatively, this behavior is clearly what we are observing in the thermal conductivity curves of the different isomers of glassy butanol.

Therefore, it seems reasonable to conclude that the SPM and its extensions provide a consistent quantitative framework to account for both the specific heat and the thermal conductivity of glasses at low temperatures, though the lack of experimental data at even lower temperatures prevents us from conducting a more incisive check for the case of butanol isomers.

In addition, the experimental mean free paths $\ell(T)$ of the heat-transporting phonons can be assessed from the standard equation of the phonon-gas kinetic theory: $\kappa(T)=$ $\frac{1}{3} v_{D} C_{V} \ell(T)$, where $C_{V}$ is the heat capacity per unit volume due to Debye acoustic phonons. Figure 7 illustrates the behavior of $\ell(T)$ for the three glassy isomers, calculated using the experimental data for the thermal conductivity. $C_{V}$ was obtained from the Debye model with $v_{D}$ taken from Table II. It can be observed that for all butanol glasses the phonon mean free path is much larger than the limiting value 
$\ell_{\min }(T)$ calculated according to the Cahill-Pohl model. This essentially harmonic model is therefore unable to account for the vibrational dynamics responsible for the thermal conductivity of glasses at low temperatures.

\section{Ioffe-Regel crossover}

Finally, the corresponding crossover energies $E_{\mathrm{IR}}$ calculated by means of the SPM Eq. (6), both for the studied butanol glasses and for other related alcohol glasses, are compared to the boson-peak maximum $T_{\max }$ of $C_{p} / T^{3}$ in Table IV and Fig. 8. As stated above, the extended SPM by Parshin, Schober, and Gurevich ${ }^{17}$ found that, approximately, $\omega^{\mathrm{IR}} \approx \omega_{\mathrm{BP}}$ and hence $E_{\mathrm{IR}} \approx 5 k_{B} T_{\max }$. For the alcohol glasses shown in Fig. 8, such a linear correlation is observed indeed, though the proportionality factor is slightly higher than 5 , finding an average value $E_{\mathrm{IR}} / k_{B} \approx 6 T_{\max }$ implying $\omega^{\mathrm{IR}} \approx 1.2 \omega_{\mathrm{BP}}$, which considering the many approximations involved is a very good agreement. One could argue that we are not truly comparing the Ioffe-Regel limit of phonon localization with the boson-peak maximum, since we have not directly measured $E_{\mathrm{IR}}$ but have rather obtained it from Eq. (7), using the SPM parameters fitting our $C_{p}(T)$ and $\kappa(T)$ data. It is nonetheless to be stressed, that a similar plot was shown by Rufflé et al. ${ }^{25}$ for different kinds of glass, including several molecular glasses such as ethanol and glycerol, but using in their case the Ioffe-Regel frequency IR measured from inelastic-X-ray-scattering (IXS) experiments and the boson-peak frequency $\omega_{\mathrm{BP}}$ from the measured vibrational density of states. Interestingly, although they discussed a good correlation $\omega^{\mathrm{IR}} \approx \omega_{\mathrm{BP}}$, one can observe a general trend where $\omega^{\mathrm{IR}}$ is slightly larger than $\omega_{\mathrm{BP}}$, especially

TABLE IV. Obtained SPM parameters, Ioffe-Regel crossover energy $E_{\mathrm{IR}}$, and temperature $T_{\max }$ of the calorimetric boson peak in $C_{p} / T^{3}$ for the studied butanol isomers and for other alcohol glasses: $\mathrm{H}$ - and D-ethanol stand for normal (i.e., hydrogenated) and for fully deuterated ethanol, respectively; SG and OG refer to structural glass and orientational glass, respectively. $E_{\mathrm{IR}}$ has been calculated by Eq. (7).

\begin{tabular}{lcccc}
\hline \hline Substance & $\begin{array}{c}W / k_{B} \\
(\mathrm{~K})\end{array}$ & $\begin{array}{c}\bar{C} \\
\left(\times 10^{-4}\right)\end{array}$ & $\begin{array}{c}E_{\mathrm{IR}} / k_{B} \\
(\mathrm{~K})\end{array}$ & $\begin{array}{c}T_{\max } \\
(\mathrm{K})\end{array}$ \\
\hline H-Ethanol, SG & $3.6^{\mathrm{a}}$ & $8.5^{\mathrm{a}}$ & 35 & $6.1^{\mathrm{b}}$ \\
H-Ethanol, OG & $3.8^{\mathrm{a}}$ & $8.2^{\mathrm{a}}$ & 37.8 & $6.8^{\mathrm{b}}$ \\
D-Ethanol, SG & $3.8^{\mathrm{a}}$ & $8.4^{\mathrm{a}}$ & 37.5 & $6.0^{\mathrm{b}}$ \\
D-Ethanol, OG & $4.1^{\mathrm{a}}$ & $9.0^{\mathrm{a}}$ & 40 & $6.4^{\mathrm{b}}$ \\
1-Propanol & $2.3^{\mathrm{c}}$ & $2.2^{\mathrm{c}}$ & 35 & $6.7^{\mathrm{b}}$ \\
2-Propanol & $2.5^{\mathrm{d}}$ & $4.5^{\mathrm{d}}$ & 30 & $5.0^{\mathrm{b}}$ \\
n-Butanol & 2.7 & 2.25 & 41.4 & 5.4 \\
sec-Butanol & 2.8 & 3.6 & 36.7 & $4.8^{\mathrm{b}}$ \\
Isobutanol & 3.1 & 3.5 & 41 & 4.8 \\
Glycerol & $4.3^{\mathrm{b}}$ & $2.3^{\mathrm{e}}$ & 65 & $8.7^{\mathrm{b}}$ \\
\hline \hline
\end{tabular}

\footnotetext{
${ }^{2}$ Reference 65.

${ }^{\mathrm{b}}$ Reference 53 .

${ }^{\mathrm{c}}$ Reference 63 .

${ }^{\mathrm{d}}$ Parameters obtained from the thermal conductivity of 2-propanol glass (Ref. 34).

e Parameters obtained from the thermal conductivity of glycerol glass (Ref. 66).
}

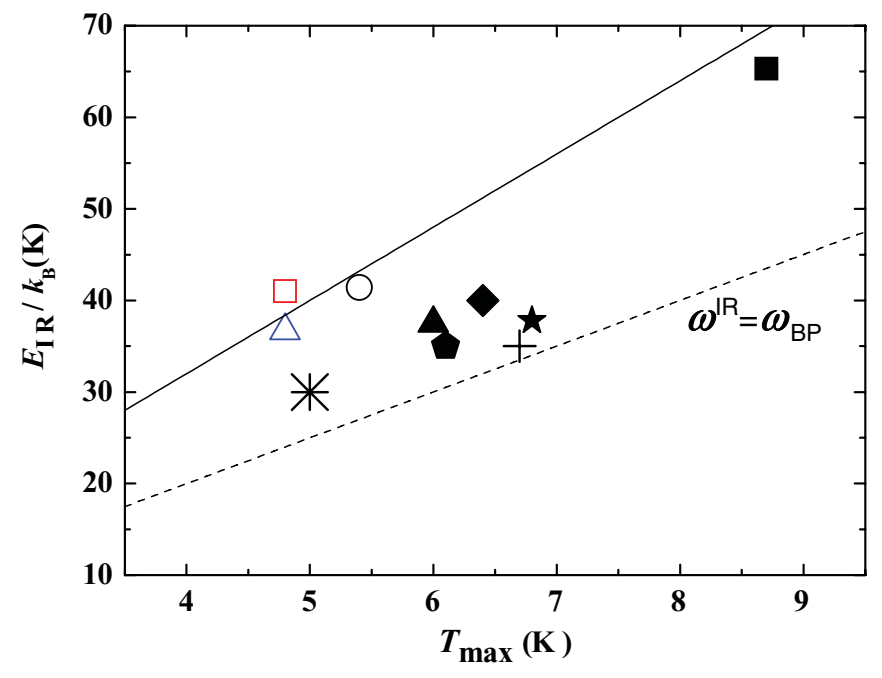

FIG. 8. (Color online) Dependence of the calculated Ioffe-Regel energy crossover $E_{\mathrm{IR}}$ on $T_{\max }$, the temperature of the calorimetric boson peak $C_{p} / T^{3}$, for different alcohol glasses (see Table IV). Butanol isomers (open symbols): $n$-butanol (black circle), sec-butanol (blue triangle), and isobutanol (red square). Other alcohols (solid symbols) are indicated as follows. Propanol isomers: 1-propanol (cross) and 2-propanol (asterisk); normal H-ethanol: structural glass (pentagon) and orientational glass (star); fully deuterated D-ethanol: structural glass (triangle) and orientational glass (lozenge); glycerol (square). Dashed and solid lines indicate possible linear correlations $E_{\mathrm{IR}}=$ $5 T_{\max }$ (corresponding roughly to $\omega^{\mathrm{IR}} \approx \omega_{\mathrm{BP}}$ ), and $E_{\mathrm{IR}}=8 T_{\max }$, respectively.

for molecular glasses and polymers, in very good agreement with our thermal data analyzed within the SPM.

Therefore, our work supports the view that the universal low-temperature properties and low-frequency dynamics of glasses (including the TLS and the boson peak) are not to be directly scaled with the elastic constants and the Debye frequency, but are only indirectly linked to the elastic medium via the Ioffe-Regel limiting frequency $\omega^{\mathrm{IR}} \approx \omega_{\mathrm{BP}}$, where lower frequency glassy quasilocalized excitations and acousticlike phonons eventually hybridize.

\section{SUMMARY AND CONCLUSIONS}

Positional isomerism in butanol glasses has been employed to shed light on our understanding of the low-temperature thermal and vibrational properties of glasses, specifically on the much controversial features known as the boson peak [in the reduced magnitudes $g(\omega) / \omega^{2}$ and $\left.C_{p} / T^{3}\right]$ and the thermal conductivity plateau. We have found that the main thermal properties of butanol glasses at low temperatures strongly vary among its different position isomers, when the hydrogen bond of the hydroxyl group $(\mathrm{OH})$ locates in a different position of the butanol molecule.

Specifically, the glass of isobutanol exhibits a $C_{p} / T^{3}$ boson-peak height which doubles that of $n$-butanol, whereas the glass of sec-butanol presents a $C_{p} / T^{3}$ peak that is $50 \%$ higher than the peak of $n$-butanol. This is in clear contrast with the elastic Debye contributions, since both the Debye specific heat of the reference crystals and the measured Debye temperatures in the same glasses from Brillouin experiments 
remain essentially constant for the different isomers. Also, the thermal conductivity at low temperatures is very different among the studied butanol isomers, though a proper scaling within the basic SPM brings all experimental data into a single master curve for $T<2 W / k_{B}$. On the contrary, the thermal conductivity becomes insensitive to the $\mathrm{OH}$ position at higher temperatures. This transition from weak resonant scattering of phonons to strong resonant scattering (i.e., the Ioffe-Regel crossover) governs the thermal conductivity of the studied glasses, making them independent of the molecular structure of the glass at higher temperatures.

Therefore, our experiments clearly contradict those works claiming (see, e.g., Refs. 22 and 55-57) a direct Debye scaling of the boson peak and hence of the excess low-temperature specific heat of glasses. However, our analysis based upon the soft-potential model and its extensions supports the proposed correlation of the boson-peak position with the Ioffe-Regel frequency $\left(\omega^{\mathrm{IR}} \approx \omega_{\mathrm{BP}}\right)$, which points out to a picture where glassy quasilocalized excitations (soft modes) and acousticlike phonons coexist as (ideally) independent entities at frequencies well below the boson peak, which marks indeed the frequency for which both kinds of vibrational excitations strongly interact and hybridize, hence piling up at those frequencies and producing a maximum in the reduced vibrational density of states.

\section{ACKNOWLEDGMENTS}

We acknowledge financial support by the Spanish Ministry of Science within program CONSOLIDER Nanociencia Molecular CSD2007-00010 and by the Comunidad de Madrid through the project NANOBIOMAGNET S2009/MAT-1726. Fruitful discussions with F. J. Bermejo and C. Cabrillo are gratefully acknowledged. *miguel.ramos@uam.es

${ }^{1}$ Amorphous Solids: Low Temperature Properties, edited by W. A. Phillips (Springer, Berlin, 1981); Rep. Prog. Phys. 50, 1657 (1987).

${ }^{2}$ P. W. Anderson, B. I. Halperin, and C. M. Varma, Philos. Mag. 25, 1 (1972); W. A. Phillips, J. Low Temp. Phys. 7, 351 (1972).

${ }^{3}$ R. O. Pohl, X. Liu, and E. J. Thompson, Rev. Mod. Phys. 74, 991 (2002).

${ }^{4}$ X. Liu and R. O. Pohl, Phys. Rev. B 58, 9067 (1998).

${ }^{5}$ C. C. Yu and A. J. Leggett, Comments Condens. Matter Phys. 14, 231 (1988).

${ }^{6}$ A. L. Burin, D. Natelson, D. D. Osheroff, and Y. Kagan, in Tunneling Systems in Amorphous and Crystalline Solids, edited by P. Esquinazi (Springer, Berlin, 1998), Chap. 5.

${ }^{7}$ J. Classen, T. Burkert, C. Enss, and S. Hunklinger, Phys. Rev. Lett. 84, 2176 (2000).

${ }^{8}$ R. König, M. A. Ramos, I. Usherov-Marshak, J. Arcas-Guijarro, A. Hernando-Mañeru, and P. Esquinazi, Phys. Rev. B 65, 180201(R) (2002).

${ }^{9}$ See, for instance, the specific sessions devoted to this topic in the Sixth International Discussion Meeting on Relaxations in Complex Systems, Rome, Italy, and their selected papers recently published in its special issue: J. Non-Cryst. Solids 357, 501 (2011).

${ }^{10}$ V. G. Karpov, M. I. Klinger, and F. N. Ignatiev, Sov. Phys. JETP 57, 439 (1983).

${ }^{11}$ M. A. Il'in, V. G. Karpov, and D. A. Parshin, Sov. Phys. JETP 65, 165 (1987).

${ }^{12}$ U. Buchenau, Yu. M. Galperin, V. L. Gurevich, and H. R. Schober, Phys. Rev. B 43, 5039 (1991).

${ }^{13}$ U. Buchenau, Yu. M. Galperin, V. L. Gurevich, D. A. Parshin, M. A. Ramos, and H. R. Schober, Phys. Rev. B 46, 2798 (1992).

${ }^{14}$ D. A. Parshin, Phys. Rev. B 49, 9400 (1994); Phys. Solid State 36, 991 (1994).

${ }^{15}$ M. A. Ramos and U. Buchenau, Phys. Rev. B 55, 5749 (1997); in Tunneling Systems in Amorphous and Crystalline Solids, edited by P. Esquinazi (Springer, Berlin, 1998), Chap. 9.

${ }^{16}$ V. L. Gurevich, D. A. Parshin, and H. R. Schober, Phys. Rev. B 67, 094203 (2003).
${ }^{17}$ D. A. Parshin, H. R. Schober, and V. L. Gurevich, Phys. Rev. B 76, 064206 (2007).

${ }^{18}$ V. Lubchenko and P. G. Wolynes, e-print arXiv:cond-mat/0506708v1, and references therein (unpublished).

${ }^{19}$ G. D’Angelo, C. Crupi, G. Tripodo, and G. Salvato, J. Phys. Chem. B 114, 2467 (2010).

${ }^{20}$ W. Schirmacher, Europhys. Lett. 73, $892 \quad$ (2006); W. Schirmacher, G. Ruocco, and T. Scopigno, Phys. Rev. Lett. 98, 025501 (2007).

${ }^{21}$ B. Rufflé, D. A. Parshin, E. Courtens, and R. Vacher, Phys. Rev. Lett. 100, 015501 (2008).

${ }^{22}$ A. I. Chumakov et al., Phys. Rev. Lett. 106, 225501 (2011).

${ }^{23}$ A. F. Ioffe and A. R. Regel, Prog. Semicond. 4, 237 (1960). Although this localization of acoustic phonons has become known as the Ioffe-Regel limit, strictly speaking this article deals with it in a somewhat different context, as has been pointed out by W. Schirmacher in Ref. 9, p. 518.

${ }^{24}$ D. A. Parshin and C. Laermans, Phys. Rev. B 63, 132203 (2001).

${ }^{25}$ B. Rufflé, G. Guimbretiére, E. Courtens, R. Vacher, and G. Monaco, Phys. Rev. Lett. 96, 045502 (2006); 98, 079602 (2007).

${ }^{26}$ U. Buchenau, A. Wischnewski, M. Ohl, and E. Fabiani, J. Phys.: Condens. Matter 19, 205106 (2007).

${ }^{27}$ H. Shintani and H. Tanaka, Nature Mater. 7, 870 (2008).

${ }^{28}$ G. Ruocco, A. Matic, T. Scopigno, and S. N. Yannopoulos, Phys. Rev. Lett. 98, 079601 (2007).

${ }^{29}$ C. Talón, M. A. Ramos, S. Vieira, G. J. Cuello, F. J. Bermejo, A. Criado, M. L. Senent, S. M. Bennington, H. E. Fischer, and H. Schober, Phys. Rev. B 58, 745 (1998).

${ }^{30}$ M. A. Ramos, C. Talón, R. J. Jiménez-Riobóo, and S. Vieira, J. Phys.: Condens. Matter 15, S1007 (2003).

${ }^{31}$ P. Nagel, A. Fleischmann, S. Hunklinger, and C. Enss, Phys. Rev. Lett. 92, 245511 (2004)

${ }^{32}$ C. Talón, M. A. Ramos, I. M. Shmyt'ko, N. Afonikova, A. Criado, G. Madariaga, and F. J. Bermejo, J. Non-Cryst. Solids 287, 226 (2001).

${ }^{33}$ C. Talón, F. J. Bermejo, C. Cabrillo, G. J. Cuello, M. A. González, J. W. Richardson Jr., A. Criado, M. A. Ramos, S. Vieira, F. L. Cumbrera, and L. M. González, Phys. Rev. Lett. 88, 115506 (2002). 
${ }^{34}$ A. I. Krivchikov, A. N. Yushchenko, O. A. Korolyuk, F. J. Bermejo, R. Fernandez-Perea, I. Bustinduy, and M. A. González, Phys. Rev. B 77, 024202 (2008).

${ }^{35}$ H. Suga and S. Seki, J. Non-Cryst. Solids 16, 171 (1974).

${ }^{36}$ M. A. Ramos, S. Vieira, F. J. Bermejo, J. Dawidowski, H. E. Fischer, H. Schober, M. A. González, C. K. Loong, and D. L. Price, Phys. Rev. Lett. 78, 82 (1997).

${ }^{37}$ C. Talón, M. A. Ramos, and S. Vieira, Phys. Rev. B 66, 012201 (2002).

${ }^{38}$ A. I. Krivchikov, A. N. Yushchenko, V. G. Manzhelii, O. A. Korolyuk, F. J. Bermejo, R. Fernández-Perea, C. Cabrillo, and M. A. González, Phys. Rev. B 74, 060201(R) (2006).

${ }^{39}$ M. A. Ramos, I. Shmyt'ko, E. A. Arnautova, R. J. Rioboo, V. Rodriguez-Mora, S. Vieira, and M. J. Capitán, J. Non-Cryst. Solids 352, 4769 (2006).

${ }^{40}$ S. Benkhof, A. Kudlik, T. Blochowicz, and E. Rössler, J. Phys.: Condens. Matter 10, 8155 (1998).

${ }^{41}$ R. Brand, P. Lukenheimer, U. Schneider, and A. Loidl, Phys. Rev. B 62, 8878 (2000).

${ }^{42}$ T. El Goresy and R. Böhmer, J. Chem. Phys. 128, 154520 (2008).

${ }^{43}$ B. Jakobsen, C. Maggi, T. Christensen, and J. Dyre, J. Chem. Phys. 129, 184502 (2008).

${ }^{44}$ P. Lukenheimer, S. Kastner, M. Köhler, and A. Loidl, Phys. Rev. E 81, 051504 (2010), and references therein.

${ }^{45}$ E. Pérez-Enciso and M. A. Ramos, Termochim. Acta 461, 50 (2007).

${ }^{46}$ A. I. Krivchikov et al., J. Low Temp. Phys. 139, 693 (2005); Phys. Chem. Chem. Phys. 7, 728 (2005).

${ }^{47}$ M. Hassaine, R. J. Jiménez-Riobóo, I. V. Sharapova, O. A. Korolyuk, A. I. Krivchikov, and M. A. Ramos, J. Chem. Phys. 131, 174508 (2009).

${ }^{48}$ I. M. Shmyt'ko, R. J. Jiménez-Riobóo, M. Hassaine, and M. A. Ramos, J. Phys.: Condens. Matter 22, 195102 (2010).

${ }^{49}$ J. K. Krüger, in Brillouin Spectroscopy and its Application to Polymers in Optical Techniques to Characterize Polymer Systems, edited by A. Bässler (Elsevier, Amsterdam, 1989).
${ }^{50}$ A. I. Krivchikov, M. Hassaine, I. V. Sharapova, O. A. Korolyuk, R. J. Jiménez-Riobóo, and M. A. Ramos, J. Non-Cryst. Solids 357, 524 (2011).

${ }^{51}$ See the discussion in the second paragraph of p. 527 in Ref. 50.

${ }^{52}$ V. O. Bodmann, Die Makromolekulare Chemie 122, 198 (1969).

${ }^{53}$ M. A. Ramos, C. Talón, and S. Vieira, J. Non-Cryst. Solids 307-310, 80 (2002).

${ }^{54}$ M. A. Ramos, Philos. Mag. 84, 1313 (2004).

${ }^{55}$ B. Ruta, G. Baldi, V. M. Giordano, L. Orsingher, S. Rols, F. Scarponi, and G. Monaco, J. Chem. Phys. 133, 041101 (2010).

${ }^{56}$ S. Caponi, S. Corezzi, D. Fioretto, A. Fontana, G. Monaco, and F. Rossi, Phys. Rev. Lett. 102, 027402 (2009).

${ }^{57}$ A. Monaco, A. I. Chumakov, Y.-Z. Yue, G. Monaco, L. Comez, D. Fioretto, W. A. Crichton, and R. Rüffer, Phys. Rev. Lett. 96, 205502 (2006).

${ }^{58}$ A. V. Granato, Phys. Rev. Lett. 68, 974 (1992); J. Phys. Chem. Solids 55, 931 (1994).

${ }^{59}$ A. V. Granato, in Non Equilibrium Phenomena in Supercooled Fluids Glasses and Amorphous Materials, edited by M. Giordano, D. Leporini, and M. P. Tosi (World Scientific, Singapore, 1996).

${ }^{60}$ G. Carini, G. D’Angelo, and G. Tripodi, Philos. Mag. 71, 539 (1995).

${ }^{61}$ X. Liu and H. V. Löhneysen, Europhys. Lett. 33, 617 (1996).

${ }^{62}$ I. V. Sharapova, A. I. Krivchikov, O. A. Korolyuk, A. Jezowski, M. Rovira-Esteva, J. L1. Tamarit, L. C. Pardo, M. D. Ruiz-Martin, and F. J. Bermejo, Phys. Rev. B 81, 094205 (2010).

${ }^{63}$ A. I. Krivchikov, O. A. Korolyuk, I. V. Sharapova, O. O. Romantsova, F. J. Bermejo, C. Cabrillo, R. Fernandez-Perea, and I. Bustinduy, J. Non-Cryst. Solids 357, 483 (2011).

${ }^{64}$ D. G. Cahill and R. O. Pohl, Phys. Rev. B 35, 4067 (1987).

${ }^{65}$ A. I. Krivchikov, F. J. Bermejo, I. V. Sharapova, O. A. Korolyuk, and O. O. Romantsova, Fis. Nizk. Temp. 37, 651 (2011).

${ }^{66}$ C. Talón, Q. W. Zou, M. A. Ramos, R. Villar, and S. Vieira, Phys. Rev. B 65, 012203 (2002). 